Claremont Colleges

Scholarship@ Claremont

All HMC Faculty Publications and Research

HMC Faculty Scholarship

$1-1-1993$

\title{
An Elementary Approach to Some Analytic Asymptotics
}

Nicholas Pippenger

Harvey Mudd College

\section{Recommended Citation}

Nicholas Pippenger. "An Elementary Approach to Some Analytic Asymptotics", Society for Industrial and Applied Mathematics Journal on Mathematical Analysis, 24, 1361 (1993).

This Article is brought to you for free and open access by the HMC Faculty Scholarship at Scholarship @ Claremont. It has been accepted for inclusion in All HMC Faculty Publications and Research by an authorized administrator of Scholarship @ Claremont. For more information, please contact scholarship@cuc.claremont.edu. 


\title{
AN ELEMENTARY APPROACH TO SOME ANALYTIC ASYMPTOTICS*
}

\author{
NICHOLAS PIPPENGER $\dagger$
}

Abstract. Fredman and Knuth have treated certain recurrences, such as $M(0)=1$ and

$$
M(n+1)=\min _{0 \leq k \leq n}(\alpha M(k)+\beta M(n-k)),
$$

where $\min (\alpha, \beta)>1$, by means of auxiliary recurrences such as

$$
h(x)= \begin{cases}0 & \text { if } 0 \leq x<1 \\ 1+h(x / \alpha)+h(x / \beta) & \text { if } 1 \leq x<\infty\end{cases}
$$

The asymptotic behavior of $h(x)$ as $x \rightarrow \infty$ with $\alpha$ and $\beta$ fixed depends on whether $\log \alpha / \log \beta$ is rational or irrational. The solution of Fredman and Knuth used analytic methods in both cases, and used in particular the Wiener-Ikehara Tauberian theorem in the irrational case. The author shows that a more explicit solution to these recurrences can be obtained by entirely elementary methods, based on a geometric interpretation of $h(x)$ as a sum of binomial coefficients over a triangular subregion of Pascal's triangle. Apart from Stirling's formula, in the irrational case only the KroneckerWeyl theorem (which can itself be proved by elementary methods) is needed, to the effect that if $\vartheta$ is irrational, the fractional parts of the sequence $\vartheta, 2 \vartheta, 3 \vartheta, \ldots$, are uniformly distributed in the unit interval.

Key words. asymptotic analysis, recurrence relation

AMS subject classification. $26 \mathrm{~A} 12$

1. Introduction. The analysis of algorithms and data structures, as well as of constructions for systems such as sorting and switching networks, often leads to recurrences. Because recursive algorithms, data structures, and constructions often involve choices that should be made in an optimal way, the recurrences often involve minimization. In their paper, Fredman and Knuth [FK] treat a large number of related recurrences by a combination of combinatorial and analytic methods. The goal of the present paper is to show how in many cases it is possible to replace the analytic component of their solutions with elementary arguments. (Here the terms "analytic" and "elementary" are used in accordance with the practice in number theory: "analytic" refers to methods based on properties of analytic functions of a complex variable, especially residues or integral transforms, while "elementary" refers to the absence of such methods. In particular, "elementary" does not refer to either simplicity or brevity.) As a bonus, we shall see that our analysis leads to a more explicit and informative solution in some cases. A preliminary version of our results appears as $[\mathrm{P}]$.

Of the recurrences treated by Fredman and Knuth, the one which best illustrates our contribution is $M(0)=1$ and

$$
M(n+1)=\min _{0 \leq k \leq n}(\alpha M(k)+\beta M(n-k)),
$$

*Received by the editors May 4, 1992; accepted for publication (in revised form) December 3, 1992. This research was partially supported by a Natural Sciences and Engineering Research Council of Canada operating grant and a British Columbia Advance Systems Institute Fellowship Award.

$\dagger$ Department of Computer Science, University of British Columbia, Vancouver, British Columbia V6T 1Z2, Canada. 
where $\alpha$ and $\beta$ are fixed parameters with $\min (\alpha, \beta)>1$. (This is the case " $g(n)=\delta_{n 0}$," dealt with in their §6.) By straightforward and elementary arguments, Fredman and Knuth reduce the study of (1.1) to that of the function $h$ defined by

$$
h(x)= \begin{cases}0 & \text { if } 0 \leq x<1 \\ 1+h(x / \alpha)+h(x / \beta) & \text { if } 1 \leq x<\infty\end{cases}
$$

The analysis of Fredman and Knuth proceeds by considering the integral transform

$$
K(s)=\int_{1}^{\infty} \frac{h(t) d t}{t^{s+1}}
$$

of $h$, which, with the aid of (1.2), can be shown to be $K(s)=1 / s\left(1-\alpha^{-s}-\beta^{-s}\right)$. This function is analytic in the open half plane $\operatorname{Re}(s)>\gamma$, where $\gamma$ is the unique real solution to

$$
\alpha^{-\gamma}+\beta^{-\gamma}=1
$$

Furthermore, $K(s)$ has a simple pole at $s=\gamma$ with residue $C=1 /\left(\alpha^{-\gamma} \log \alpha^{\gamma}+\right.$ $\left.\beta^{-\gamma} \log \beta^{\gamma}\right)$, as is easily calculated. This pole will ultimately give rise to a factor $C x^{\gamma}$ in the asymptotic behavior of $h(x)$.

The behavior of $K(s)$ on the remainder of the critical line $\operatorname{Re}(s)=\gamma$ depends on whether $\log \alpha / \log \beta$ is rational or irrational. If this quotient is irrational, the pole at $s=\gamma$ is the only one on the critical line, and a Tauberian theorem due to Wiener, Ikehara, and Landau (Lemma 4.3 in Fredman and Knuth) leads to the conclusion that

$$
h(x) \sim C x^{\gamma}
$$

in this case. If the quotient $\log \alpha / \log \beta$ is rational, $K(s)$ has additional poles periodically disposed along the critical line. Application of Cauchy's residue theorem leads to the conclusion that

$$
h(x) \sim D(x) x^{\gamma}
$$

in this case, where $D(x)$ is a periodic function of $\log x$ whose period is determined by the spacing between poles along the critical line, and whose Fourier coefficients are determined by the residues at those poles.

In this paper we shall derive (1.4) and (1.5) in an elementary fashion. This new derivation has the merit of giving a simple explicit formula for the function $D(x)$ in (1.5). We shall also want the solution to the related recurrence

$$
h^{\prime}(x)= \begin{cases}0 & \text { if } 0 \leq x<1 \\ 1+\alpha h(x / \alpha)+\beta h(x / \beta) & \text { if } 1 \leq x<\infty\end{cases}
$$

By analogous elementary methods, we shall show that

$$
h^{\prime}(x) \sim C^{\prime} x^{\gamma+1}
$$

where $C^{\prime}=1 /\left(\alpha^{-\gamma} \log \alpha^{\gamma+1}+\beta^{-\gamma} \log \beta^{\gamma+1}\right)$ in the irrational case, and

$$
h^{\prime}(x) \sim D^{\prime}(x) x^{\gamma+1}
$$


where $D^{\prime}(x)$ is a periodic function of $\log x$ which will be determined explicitly in the rational case.

Fredman and Knuth showed that (1.4) implies that

$$
M(n) \sim A n^{1+1 / \gamma},
$$

where $A$ is an explicitly determined constant in the irrational case. We shall show that (1.5) and (1.8) together imply

$$
M(n) \sim B(n) n^{1+1 / \gamma},
$$

where $B(n)$ is an explicitly determined periodic function of $\log n$ in the rational case. (Once the form of the functions $D(x)$ and $D^{\prime}(x)$ are explicitly known, it is possible to go back and derive these results by extending the analysis of Fredman and Knuth. This would involve showing that certain Fourier series converge to certain periodic functions. But since there is no general procedure for identifying a function from its Fourier series, it does not appear to be possible to extend the analysis of Fredman and Knuth without knowing what $D(x)$ and $D^{\prime}(x)$ are by some other method.)

2. The rational case. Our analysis begins with the observation that $h(x)$ is the number of words over the alphabet $\{\alpha, \beta\}$ having weight at most $x$, where the weight of a word is the product of its letters. (We take the weight of the empty word to be unity.) Indeed, if $0 \leq x<1$, then there are no such words and $h(x)=0$. If $1 \leq x<\infty$, then $h(x)=1+h(x / \alpha)+h(x / \beta)$, and any word for which the product of the letters is at most $x$ must either be empty (and there is 1 such word) or consist of an $\alpha$ followed by a word for which the product of the letters is at most $x / \alpha$ (and there are $h(x / \alpha)$ such words), or consist of an $\beta$ followed by a word for which the product of the letters is at most $x / \beta$ (and there are $h(x / \beta)$ such words). Since there are exactly $\left(\begin{array}{c}i+j \\ i\end{array}\right)$ words that contain $i \alpha$ 's and $j \beta$ 's, we have established the following explicit formula for $h(x)$ :

$$
h(x)=\sum_{\alpha^{i} \beta^{j} \leq x}\left(\begin{array}{c}
i+j \\
i
\end{array}\right) .
$$

Taking logarithms in the constraint of the summation, we see that $h(x)$ may be interpreted as the sum of the binomial coefficients $\left(\begin{array}{c}i+j \\ i\end{array}\right)$ in Pascal's triangle over the triangular subregion bounded by the inequalities $i \geq 0, j \geq 0$, and

$$
i \log \alpha+j \log \beta \leq \log x .
$$

Suppose that $\log \alpha / \log \beta$ is the rational number $p / q$, where $p$ and $q$ are positive integers such that $\operatorname{gcd}(p, q)=1$. Then $\log _{\alpha \beta} \alpha=p /(p+q), \log _{\alpha \beta} \beta=q /(p+q)$, and if we set

$$
\varrho=(\alpha \beta)^{1 /(p+q)}
$$

then (2.2) becomes

$$
p i+q j \leq \log _{\varrho} x .
$$

Since $p, q, i$, and $j$ are integers, we see that $h(x)$ remains constant as $x$ increases except when $\log _{\varrho} x$ passes through an integer $k$ when it jumps by

$$
S(k)=\sum_{p i+q j=k}\left(\begin{array}{c}
i+j \\
i
\end{array}\right) .
$$


We shall see below that $S(k)$ has the asymptotic formula

$$
S(k) \sim(C \log \sigma) \sigma^{k}
$$

where

$$
\sigma=\varrho^{\gamma}=(\alpha \beta)^{\gamma /(p+q)} .
$$

If we set $S^{*}(l)=\sum_{0 \leq k \leq l} S(k)$, it follows that

$$
S^{*}(l) \sim \frac{(C \sigma \log \sigma) \sigma^{l}}{\sigma-1}
$$

This formula gives the asymptotic value $S^{*}(l)$ of $h(x)$ when $x$ is a "magic" number of the form $x=\varrho^{l}$. The asymptotic formula for arbitrary $x$ follows from this and the fact that $h(x)$ remains constant between magic values of $x$. If we write $\log _{\varrho} x=l+\lambda$, where $l=\left\lfloor\log _{\varrho} x\right\rfloor$ (the integral part of $\log _{\varrho} x$ ) and $\lambda=\left\{\log _{\varrho} x\right\}$ (the fractional part of $\left.\log _{\varrho} x\right)$, then

$$
\begin{aligned}
h(x) & \sim \frac{(C \sigma \log \sigma) \sigma^{l}}{\sigma-1} \\
& \sim \frac{\left(C \sigma^{1-\lambda} \log \sigma\right) \sigma^{l+\lambda}}{\sigma-1} \\
& \sim P\left(\left\{\log _{\varrho} x\right\}\right) x^{\gamma}
\end{aligned}
$$

where

$$
P(\lambda)=\frac{C \sigma^{1-\lambda} \log \sigma}{\sigma-1} .
$$

This establishes (1.5) with $D(x)=P\left(\left\{\log _{\varrho} x\right\}\right)$, which is periodic in $\log x$ (with period $\log \varrho$ ), as claimed.

It remains to establish (2.4). The major steps of the derivation are as follows. First, we approximate the binomial coefficients $\left(\begin{array}{c}i+j \\ i\end{array}\right)=(i+j) ! / i ! j !$ in $(2.3)$ by applying Stirling's formula to their constituent factorials. If we separate the approximation into algebraically varying factors and exponentially varying factors, we see that the exponentially varying factors impart to the summand a peaking reminiscent of the central limit theorem: the greatest contribution to the sum comes when $i$ and $j$ are in

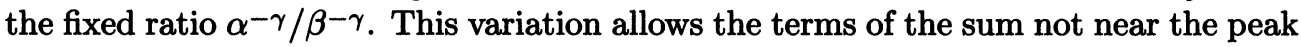
to be neglected. The resulting truncated sum is then estimated by an integral; the error in this estimation is at most the total variation of the summand, which (since the summand is unimodal) is at most twice the largest term. The resulting integral can be transformed into the well-known integral $\int_{-\infty}^{+\infty} e^{-y^{2}} d y=\pi^{1 / 2}$ by adjoining negligible tails. The result is (2.4).

Successive values of $i$ and $j$ differ by $q$ and $p$, respectively; it will be convenient to have an index whose successive values differ by 1 . Thus we introduce the index $m$ satisfying

$$
i=q m, \quad j=k / q-p m, \quad i+j=k / q-(p-q) m .
$$

This index assumes values that are not necessarily integers, but are congruent to $1 / q$ modulo 1.

LEMMA 2.1.

$$
\left(\begin{array}{c}
i+j \\
i
\end{array}\right)=\left(1+O\left(\frac{1}{i}+\frac{1}{j}\right)\right)\left(\frac{i+j}{2 \pi i j}\right)^{1 / 2} \exp k E\left(\frac{m}{k}\right)
$$


where

$$
\begin{aligned}
& E(\mu)=F(G(\mu)), \quad F(\nu)=\frac{H(\nu)}{p \nu+q(1-\nu)}, \\
& G(\mu)=\frac{q^{2} \mu}{1-(p-q) q \mu}, \quad H(\nu)=-\nu \log \nu-(1-\nu) \log (1-\nu) .
\end{aligned}
$$

Proof. The estimate

$$
\left(\begin{array}{c}
i+j \\
i
\end{array}\right)=\left(1+O\left(\frac{1}{i}+\frac{1}{j}\right)\right)\left(\frac{i+j}{2 \pi i j}\right)^{1 / 2} \exp (i+j) H\left(\frac{i}{i+j}\right)
$$

is an immediate consequence of Stirling's formula

$$
n !=\left(1+O\left(\frac{1}{n}\right)\right)(2 \pi n)^{1 / 2} e^{-n} n^{n}
$$

(see Knuth $[K 1, \S 1.2 .11])$. Define $\nu$ such that

$$
i=\nu(i+j), \quad j=(1-\nu)(i+j), \quad k=(p \nu+q(1-\nu))(i+j) .
$$

Then

$$
\left(\begin{array}{c}
i+j \\
i
\end{array}\right)=\left(1+O\left(\frac{1}{i}+\frac{1}{j}\right)\right)\left(\frac{i+j}{2 \pi i j}\right)^{1 / 2} \exp k F\left(\frac{i}{i+j}\right) .
$$

Define $\mu$ such that

$$
m=\mu k \text {. }
$$

Then $\nu$ and $\mu$ are related by

$$
\nu=\frac{q^{2} \mu}{1-(p-q) q \mu}, \quad \mu=\frac{\nu}{q^{2}+(p-q) q \nu} .
$$

This yields the assertion of the lemma.

LEMMA 2.2. The function $F(\nu)$ assumes its unique maximum (for $0 \leq \nu \leq 1$ ) at

$$
N=\sigma^{-p} .
$$

At this point

$$
F(N)=\log \sigma, \quad F^{\prime}(N)=0, \quad F^{\prime \prime}(N)=-\frac{1}{N(1-N) \Delta}
$$

where

$$
\Delta=p N+q(1-N)
$$

and the primes indicate differentiation. Accordingly, $E(\mu)$ assumes its maximum at

$$
M=\frac{N}{q \Delta}
$$

and at this point

$$
E(M)=\log \sigma, \quad E^{\prime}(M)=0, \quad E^{\prime \prime}(M)=\frac{\Delta^{3}}{N(1-N)}
$$


Proof. We shall let $H(0)=H(1)=0$; this makes $H(\nu)$, and therefore $F(\nu)$, continuous on the closed interval $0 \leq \nu \leq 1$. These functions are in fact analytic in the open interval $0<\nu<1$, and thus $F(\nu)$ can assume its maximum only where its first derivative vanishes or at an endpoint. We compute the first derivatives

$$
\begin{aligned}
& H^{\prime}(\nu)=\log (1-\nu)-\log \nu \\
& F^{\prime}(\nu)=-\frac{H(\nu)(p-q)}{(p \nu+q(1-\nu))^{2}}+\frac{H^{\prime}(\nu)}{p \nu+q(1-\nu)} .
\end{aligned}
$$

Equating $F^{\prime}(\nu)$ to zero leads to the equation

$$
(p \nu+q(1-\nu)) H^{\prime}(\nu)=(p-q) H(\nu) .
$$

This has the unique solution $\nu=N$, where

$$
N=\alpha^{-\gamma}=\sigma^{-p}
$$

so that

$$
1-N=\beta^{-\gamma}=\sigma^{-q} \text {. }
$$

This gives

$$
F(N)=\log \sigma
$$

which is obviously larger than $F(\nu)$ at either of the endpoints. We compute the second derivatives

$$
\begin{aligned}
& H^{\prime \prime}(\nu)=-\frac{1}{\nu(1-\nu)} \\
& F^{\prime \prime}(\nu)=\frac{2 H(\nu)(p-q)^{2}}{(p \nu+q(1-\nu))^{3}}-\frac{2 H^{\prime}(\nu)(p-q)}{(p \nu+q(1-\nu))^{2}}+\frac{H^{\prime \prime}(\nu)}{p \nu+q(1-\nu)} .
\end{aligned}
$$

Since the first two terms of $F^{\prime \prime}(\nu)$ are a multiple of $F^{\prime}(\nu)$, they vanish at $\nu=N$, leaving

$$
F^{\prime \prime}(N)=-\frac{1}{N(1-N) \Delta}
$$

This derivation can be carried over to $E(\mu), E^{\prime}(\mu)$, and $E^{\prime \prime}(\mu)$ through the derivative

$$
G^{\prime}(\mu)=\frac{q^{2}}{(1-(p-q) q \mu)^{2}}=(p \nu+q(1-\nu))^{2}
$$

and the chain rule.

LEMMA 2.3.

$$
\begin{aligned}
& \sum_{m}\left(1+O\left(\frac{1}{i}+\frac{1}{j}\right)\right)\left(\frac{i+j}{2 \pi i j}\right)^{1 / 2} \exp k E\left(\frac{m}{k}\right) \\
& =\left(1+O\left(\frac{(\log k)^{3 / 2}}{k^{1 / 2}}\right)\right) \frac{\sigma^{k}}{\Delta}
\end{aligned}
$$

Here the sum over $m$, as described above, is over $i \geq 0$ and $j \geq 0$ such that $i+j=k$, with $m=i / q$. 
Proof. The major steps of the derivation are as follows. The central peaking of the summand will be exploited, allowing the tails of the summation to be neglected. The decaudated sum can be simplified, since the algebraically varying factors behave like constants in the remaining range of summation. The resulting sum will be estimated with an integral, to which the tails previously removed will be restored. The recaudated integral can be evaluated by standard methods.

Our sum is

$$
\sum_{m} W_{m}
$$

where

$$
W_{m}=\left(1+O\left(\frac{1}{i}+\frac{1}{j}\right)\right)\left(\frac{i+j}{2 \pi i j}\right)^{1 / 2} \exp k E\left(\frac{m}{k}\right) .
$$

Since $E(\mu)$ is analytic at $\mu=M$, it can be expanded in a Taylor series about this point. The result is

$$
E(\mu)=\log \sigma-(\mu-M)^{2} / \delta^{2}+O\left((\mu-M)^{3}\right)
$$

where

$$
\delta=\left(\frac{2 N(1-N)}{\Delta^{3}}\right)^{1 / 2} .
$$

We shall break our sum into three parts,

$$
\sum_{m} W_{m}=\sum_{m<a} W_{m}+\sum_{a \leq m \leq b} W_{m}+\sum_{b<m} W_{m}
$$

where

$$
\begin{aligned}
& a=M k-\left(\frac{6 N(1-N) k \log k}{\Delta^{3}}\right)^{1 / 2}, \\
& b=M k+\left(\frac{6 N(1-N) k \log k}{\Delta^{3}}\right)^{1 / 2} .
\end{aligned}
$$

To estimate the sum over $m<a$, we observe that it comprises $O(k)$ terms, each of which is at most $W_{a}$. We have $E(a / k)=\log \sigma-3(\log k / k)+O\left((\log k / k)^{3 / 2}\right)$ (by the Taylor expansion). Thus

$$
W_{a}=O\left(\frac{\sigma^{k}}{k^{3}}\right)
$$

and so

$$
\sum_{m<a} W_{m}=O\left(\frac{\sigma^{k}}{k^{2}}\right)
$$

Similarly,

$$
\sum_{b<m} W_{m}=O\left(\frac{\sigma^{k}}{k^{2}}\right)
$$

and thus

$$
\sum_{m} W_{m}=\sum_{a \leq m \leq b} W_{m}+O\left(\frac{\sigma^{k}}{k^{2}}\right)
$$


For any term in the sum over $a \leq m \leq b$,

$$
m=M k\left(1+O\left(\left(\frac{\log k}{k}\right)^{1 / 2}\right)\right)
$$

from which it follows that

$$
\begin{aligned}
& i=\frac{N k}{\Delta}\left(1+O\left(\left(\frac{\log k}{k}\right)^{1 / 2}\right)\right), \\
& j=\frac{(1-N) k}{\Delta}\left(1+O\left(\left(\frac{\log k}{k}\right)^{1 / 2}\right)\right),
\end{aligned}
$$

and

$$
i+j=\frac{k}{\Delta}\left(1+O\left(\left(\frac{\log k}{k}\right)^{1 / 2}\right)\right)
$$

Thus

$$
W_{m}=\left(1+O\left(\frac{(\log k)^{3 / 2}}{k^{1 / 2}}\right)\right)\left(\frac{\Delta}{2 \pi k N(1-N)}\right)^{1 / 2} \sigma^{k} V_{m}
$$

where

$$
V_{m}=\exp -\left((m-M k)^{2} / \delta^{2} k\right)
$$

and therefore

$$
\sum_{a \leq m \leq b} W_{m}=\left(1+O\left(\frac{(\log k)^{3 / 2}}{k^{1 / 2}}\right)\right)\left(\frac{\Delta^{3}}{2 \pi k N(1-N)}\right)^{1 / 2} \sigma^{k} \sum_{a \leq m \leq b} V_{m} .
$$

Now,

$$
\sum_{a \leq m \leq b} V_{m}=\int_{a}^{b} V_{x} d x+O(1)
$$

since the total variation of the integrand is $O(1)$. We shall express our integral as the sum of three integrals:

$$
\int_{a}^{b} V_{x} d x=-\int_{-\infty}^{a} V_{x} d x+\int_{-\infty}^{+\infty} V_{x} d x-\int_{a}^{+\infty} V_{x} d x
$$

Integration by parts gives

$$
\int_{-\infty}^{a} V_{x} d x=O\left(\frac{V_{a}}{a}\right)=O\left(\frac{1}{k^{4}}\right) .
$$

Similarly

$$
\int_{a}^{+\infty} V_{x} d x=O\left(\frac{1}{k^{4}}\right)
$$

and thus

$$
\int_{a}^{b} V_{x} d x=\int_{-\infty}^{+\infty} V_{x} d x+O\left(\frac{1}{k^{4}}\right)
$$


Using the transformation

$$
x=M k+\delta k^{1 / 2} y
$$

and the well-known integral

$$
\int_{-\infty}^{+\infty} \exp -y^{2} d y=\pi^{1 / 2}
$$

we obtain

$$
\int_{-\infty}^{+\infty} V_{x} d x=\left(\frac{2 \pi k N(1-N)}{\Delta^{3}}\right)^{1 / 2} .
$$

Working backwards through (2.8), (2.7), (2.6), and (2.5) yields the assertion of the lemma.

The formula (2.4) follows from Lemma 2.3, since $C \log \sigma=1 / \Delta$.

We should mention here that the special cases $p=q=1$, where $S(k)=2^{k}$, and $p=1, q=2$, where $S(k)=F_{k+1} \sim \phi^{k+1} / \sqrt{5}$ (in which $F_{n}$ is the $n$th Fibonacci number and $\phi=(1+\sqrt{5}) / 2$; see Knuth [K1, 11.2 .8 , eq. (15) and Exer. 16]) are well known, and the analysis just given can be regarded as a generalization of these cases. Furthermore, that $\sigma=2$ and $\sigma=\phi$ are algebraic in the examples just cited is not accidental: the rationality of $\log \alpha / \log \beta=p / q$ implies that $\alpha^{-\gamma}$ and $\beta^{-\gamma}$ are roots of the polynomials $(1-z)^{p}=z^{q}$ and $z^{p}=(1-z)^{q}$, respectively, whence $\sigma=(\alpha \beta)^{\gamma /(p+q)}$ is algebraic.

In $\S 4$ we shall also want the solution to the recurrence (1.6) for $h^{\prime}(x)$ in the rational case. Let us call the product of the letters in a word over the alphabet $\{\alpha, \beta\}$ the weight of the word. Then $h^{\prime}(x)$ is the sum of the weights of all words whose weight is at most $x$, and thus we have the explicit formula

$$
h^{\prime}(x)=\sum_{\alpha^{i} \beta^{j} \leq x}\left(\begin{array}{c}
i+j \\
i
\end{array}\right) \alpha^{i} \beta^{j} .
$$

The treatment of this sum is completely analogous to that of (2.1); the result is

$$
h^{\prime}(x) \sim P^{\prime}\left(\left\{\log _{\varrho} x\right\}\right) x^{\gamma+1},
$$

where

$$
P^{\prime}(\lambda)=\frac{C^{\prime} \tau^{1-\lambda} \log \tau}{\tau-1}
$$

in which

$$
\tau=\varrho^{\gamma+1}=(\alpha \beta)^{(\gamma+1) /(p+q)} .
$$

This establishes $(1.8)$ with $D^{\prime}(x)=P^{\prime}\left(\left\{\log _{\varrho} x\right\}\right)$.

3. The irrational case. When $\log \alpha / \log \beta$ is irrational the analysis of the preceding section is not applicable, for as $x$ increases new binomial coefficients enter the sum one by one, rather than in the regularly spaced platoons of the rational case. Furthermore, the order of their entry is very irregular, with small coefficients near the axes being interspersed with large ones near the main diagonal. The analysis of this section is based on a regularity of averages amid this irregularity of detail, as expressed by the "ergodicity of an irrational rotation of the circle." We shall use in particular the Kronecker-Weyl theorem, to the effect that if $\vartheta$ is irrational, then the 
fractional parts of the sequence $\vartheta, 2 \vartheta, 3 \vartheta, \ldots$, are uniformly distributed in the unit interval. (This theorem as stated was proved by Weyl [W1]; Kronecker [K2] proved that if $\vartheta$ is irrational, then the fractional parts of the sequence $\vartheta, 2 \vartheta, 3 \vartheta, \ldots$ are dense in the unit interval.) Weyl's orginal proof (which is probably still the simplest proof) of this theorem was based on Fourier series, which by some tastes might not be accepted as elementary. A subsequent proof based on continued fractions (see Nivin [N, Chap. $6, \S 3])$ is incontestably elementary, however.

We shall say that a subset $\Xi$ of the unit interval is an "interval modulo 1 " if it is the image modulo 1 of an interval. (Thus $[0, \xi / 2) \cup[1-\xi / 2,1$ ) is an interval modulo 1 of length $\xi$.) The Kronecker-Weyl theorem asserts the following.

Let $\vartheta$ be irrational. For every $0<\xi<1$ and $0<\eta<1$, there exists a natural number $t$ such that, if $\Xi$ is an interval modulo 1 of length $\xi$ and $T$ is any set of $t$ consecutive integers, then at least $(1-\eta) \xi t$ and at most $(1+\eta) \xi t$ of the integers $i$ in $T$ are such that $\{i \vartheta\}$ falls in $\Xi$. (This theorem is often stated in the special case in which $T=\{1, \ldots, t\}$, but shifting $T$ to $T+u$ is equivalent to shifting $\Xi$ to $\Xi-u \vartheta$ modulo 1 , so the special case implies the general.)

Let $\varepsilon>0$ be fixed. Define the function $h_{\varepsilon}(x)$ by

$$
h_{\varepsilon}(x)=\sum_{x e^{-\varepsilon<\alpha^{i} \beta^{j} \leq x}}\left(\begin{array}{c}
i+j \\
i
\end{array}\right) .
$$

Taking logarithms in the constraint of the summation, we see that $h_{\varepsilon}(x)$ may be interpreted as the sum of the binomial coefficient over the trapezoidal region bounded by the inequalities $i \geq 0, j \geq 0$, and

$$
\log x-\varepsilon<i \log \alpha+j \log \beta \leq \log x .
$$

We shall see below that $h_{\varepsilon}(x)$ satisfies the asymptotic inequalities

$$
C \gamma \varepsilon(1-\varepsilon) e^{-\gamma \varepsilon} x^{\gamma} \lesssim h_{\varepsilon}(x) \lesssim C \gamma \varepsilon(1+\varepsilon) x^{\gamma}
$$

as $x \rightarrow \infty$ with $\varepsilon$ fixed. (Here $f(x) \lesssim g(x)$ means that $\lim _{\sup _{x \rightarrow \infty}} f(x) / g(x) \leq 1$.) If we set $l=\lfloor\log x / \varepsilon\rfloor+1$, then $x e^{-l \varepsilon}<1$, so we have

$$
h(x)=\sum_{0 \leq k \leq l} h_{\varepsilon}\left(x e^{-k \varepsilon}\right) .
$$

It follows that

$$
\frac{C \gamma \varepsilon(1-\varepsilon) e^{-\gamma \varepsilon} x^{\gamma}}{1-e^{-\gamma \varepsilon}} \lesssim h(x) \lesssim \frac{C \gamma \varepsilon(1+\varepsilon) x^{\gamma}}{1-e^{-\gamma \varepsilon}} .
$$

Since this holds for every $\varepsilon>0$, we may let $\varepsilon \rightarrow 0$ and obtain (1.4).

It remains to establish (3.3). The proof follows the same general lines as that of (2.4), but is complicated by the fact that the lattice points $(i, j)$ are not equally spaced in the trapezoid (3.2) as they were along the boundary of the triangle (2.2). Our salvation comes from the Kronecker-Weyl theorem, which shows that though they are not "equally spaced," they are "uniformly distributed." This will allow the trapezoid (3.2) to be broken into pieces, each of which is sufficiently large so that it contains a number of lattice points approximately proportional to its area, yet sufficiently small so that the binomial coefficients associated with these lattice points are approximately equal. 
Suppose that $\varepsilon<\log \beta$ and set $\vartheta=\log \alpha / \log \beta$, so that $\vartheta$ is irrational. Let us say that a natural number $i$ is "lucky" if there exists a $j$ such that $i$ and $j$ satisfy the inequalities (3.2). Clearly there exists such a $j$ if and only if $\{i \vartheta\}$ falls in the interval $((\log x-\varepsilon) / \log \beta, \log x / \log \beta]$. (Since the length of this interval is $\varepsilon / \log \beta<1$, it contains either no integers or one integer.) By the Kronecker-Weyl theorem, with $\xi=\varepsilon / \log \beta$ and $\eta=\varepsilon$, we may choose $t$ such that among any $t$ consecutive natural numbers there are $(1 \pm \varepsilon) \varepsilon t / \log \beta$ lucky values of $i$.

For lucky $i$, we shall regard $j$ as a function of $i$. We shall abbreviate $i \log \alpha+j \log \beta$ by $k$ (which is not necessarily an integer). We shall abbreviate $\log x$ by $l$ (which is not necessarily an integer), and $l-k$ by $\lambda$ (so that $0 \leq \lambda<\varepsilon$ ).

Since we no longer have the parameters $p$ and $q$, we shall use $\log \alpha$ and $\log \beta$ in their stead. Thus we introduce $m$ satisfying

$i=m \log \beta, \quad j=(l-\lambda) / \log \beta-m \log \alpha, \quad i+j=(l-\lambda) / \log \beta-m(\log \alpha-\log \beta)$.

Let us say that a value of $m$ is "lucky" if it corresponds to a lucky value of $i$. Henceforth, we shall take $m$ to range over lucky values, and regard $i, j, k$, and $\lambda$ as functions of $m$ for these lucky values.

By analogy with Lemma 2.1, we have

$$
\left(\begin{array}{c}
i+j \\
i
\end{array}\right)=\left(1+O\left(\frac{1}{i}+\frac{1}{j}\right)\right)\left(\frac{i+j}{2 \pi i j}\right)^{1 / 2} \exp k E\left(\frac{m}{k}\right)
$$

where

$$
\begin{aligned}
& E(\mu)=F(G(\mu)), \quad F(\nu)=\frac{H(\nu)}{\nu \log \alpha+(1-\nu) \log \beta}, \\
& G(\mu)=\frac{\mu(\log \beta)^{2}}{1-(\log \alpha-\log \beta) \log \beta}, \quad H(\nu)=-\nu \log \nu-(1-\nu) \log (1-\nu) .
\end{aligned}
$$

By analogy with Lemma 2.2, the function $F(\nu)$ assumes its unique maximum (for $0 \leq \nu \leq 1)$ at

$$
N=\alpha^{-\gamma}, \quad 1-N=\beta^{-\gamma} .
$$

At this point

$$
F(N)=\gamma, \quad F^{\prime}(N)=0, \quad F^{\prime \prime}(N)=-\frac{1}{N(1-N) \Delta},
$$

where

$$
\Delta=N \log \alpha+(1-N) \log \beta .
$$

Accordingly, $E(\mu)$ assumes its maximum at

$$
M=\frac{N}{\Delta \log \beta},
$$

and at this point

$$
E(M)=\gamma, \quad E^{\prime}(M)=0, \quad E^{\prime \prime}(M)=\frac{\Delta^{3}}{N(1-N)} .
$$

We now seek the analog of Lemma 2.3, which is the following. 
LEMMA 3.1.

$$
\begin{aligned}
(1 & \left.+O\left(\frac{(\log l)^{3 / 2}}{l^{1 / 2}}\right)\right) \frac{(1-\varepsilon) e^{-\gamma \varepsilon} \varepsilon x^{\gamma}}{\Delta} \\
& \leq \sum_{m}\left(1+O\left(\frac{1}{i}+\frac{1}{j}\right)\right)\left(\frac{i+j}{2 \pi i j}\right)^{1 / 2} \exp k E\left(\frac{m}{k}\right) \\
& \leq\left(1+O\left(\frac{(\log l)^{3 / 2}}{l^{1 / 2}}\right)\right) \frac{(1+\varepsilon) \varepsilon x^{\gamma}}{\Delta}
\end{aligned}
$$

In this lemma and all that follows, the constants ,implicit in the O-terms may depend on $\varepsilon$. The inequalities involving the $O$-terms are to be interpreted as follows: for every $\varepsilon>0$ and every choice of the $O$-term in the middle expression, there exist choices of the $O$-terms in the outer expression such that the inequalities are satisfied for all $x$.

Proof. Since $l-\varepsilon \leq k \leq l$ and $E(\mu) \leq \gamma$, we have

$$
e^{-\gamma \varepsilon} \exp l E\left(\frac{m}{k}\right) \leq \exp k E\left(\frac{m}{k}\right) \leq \exp l E\left(\frac{m}{k}\right) .
$$

Thus it will suffice to prove

$$
\begin{aligned}
(1 & \left.+O\left(\frac{(\log l)^{3 / 2}}{l^{1 / 2}}\right)\right) \frac{(1-\varepsilon) \varepsilon x^{\gamma}}{\Delta} \\
& \leq \sum_{m}\left(1+O\left(\frac{1}{i}+\frac{1}{j}\right)\right)\left(\frac{i+j}{2 \pi i j}\right)^{1 / 2} \exp l E\left(\frac{m}{k}\right) \\
& \leq\left(1+O\left(\frac{(\log l)^{3 / 2}}{l^{1 / 2}}\right)\right) \frac{(1+\varepsilon) \varepsilon x^{\gamma}}{\Delta}
\end{aligned}
$$

Choose $t$ using the Kronecker-Weyl theorem so that, for any interval of length $\varepsilon / \log \beta$ modulo 1 , among any $t$ consecutive integers $i$, there are between $(1-\varepsilon) \varepsilon t / \log \beta$ and $(1+\varepsilon) \varepsilon t / \log \beta$ such that $\{i \vartheta\}$ falls in the given interval modulo 1 . Set $L=$ $(t \log \beta) / 2$. Set

$$
Q=\left(\frac{6 N(1-N) l \log l}{\Delta^{3}}\right)^{1 / 2}
$$

and set

$$
q=\left\lceil\frac{Q-L}{2 L}\right\rceil
$$

so that $(2 q+1) L$ is the smallest odd multiple of $L$ that is not less than $Q$.

Our sum is

$$
\sum_{m} W_{m}
$$

where

$$
W_{m}=\left(1+O\left(\frac{1}{i}+\frac{1}{j}\right)\right)\left(\frac{i+j}{2 \pi i j}\right)^{1 / 2} \operatorname{expl}\left(\frac{m}{k}\right) .
$$

Since $E(\mu)$ is analytic at $\mu=M$, it can be expanded in a Taylor series about this point. The result is

$$
E(\mu)=\gamma-(\mu-M)^{2} / \delta^{2}+O\left((\mu-M)^{3}\right)
$$


where

$$
\delta=\left(\frac{2 N(1-N)}{\Delta^{3}}\right)^{1 / 2}
$$

We shall break our sum into three parts,

$$
\sum_{m} W_{m}=\sum_{m<a} W_{m}+\sum_{a \leq m<b} W_{m}+\sum_{b \leq m} W_{m}
$$

where

$$
\begin{aligned}
& a=M l-(2 q+1) L, \\
& b=M l+(2 q+1) L .
\end{aligned}
$$

We shall need the following approximation property of $E(\mu)$. If $m$ is of the form

$$
m=M l+O\left((l \log l)^{1 / 2}\right),
$$

then (since $|k-l| \leq \varepsilon$ ) we have

$$
\left|\frac{m}{k}-\frac{m}{l}\right|=O\left(\frac{1}{l}\right)
$$

Furthermore, since $E^{\prime}(\mu)=O(|\mu-M|)$ in a fixed neighborhood of $M$, we have

$$
\left|E\left(\frac{m}{k}\right)-E\left(\frac{m}{l}\right)\right|=O\left(\frac{(\log l)^{1 / 2}}{l^{3 / 2}}\right) .
$$

To estimate the sum over $m<a$, we observe that it comprises $O(l)$ terms, each of which is at most $W_{a}$. We have $E(a / k)=E(a / l)+O\left((\log l)^{1 / 2} / l^{3 / 2}\right)$ (by the approximation property with $m=a)$ and $E(a / l)=\gamma-3(\log l / l)+O\left((\log l / l)^{3 / 2}\right)$ (by the Taylor series expansion). Thus

$$
W_{a}=O\left(\frac{x^{\gamma}}{l^{3}}\right)
$$

and so

$$
\sum_{m<a} W_{m}=O\left(\frac{x^{\gamma}}{l^{2}}\right)
$$

Similarly,

$$
\sum_{b \leq m} W_{m}=O\left(\frac{x^{\gamma}}{l^{2}}\right)
$$

and thus

$$
\sum_{m} W_{m}=\sum_{a \leq m<b} W_{m}+O\left(\frac{x^{\gamma}}{l^{2}}\right) .
$$

For any term in the sum over $a \leq m<b$,

$$
m=M l\left(1+O\left(\left(\frac{\log l}{l}\right)^{1 / 2}\right)\right),
$$


from which it follows that

$$
\begin{aligned}
& i=\frac{N l}{\Delta}\left(1+O\left(\left(\frac{\log l}{l}\right)^{1 / 2}\right)\right), \\
& j=\frac{(1-N) l}{\Delta}\left(1+O\left(\left(\frac{\log l}{l}\right)^{1 / 2}\right)\right),
\end{aligned}
$$

and

$$
i+j=\frac{l}{\Delta}\left(1+O\left(\left(\frac{\log l}{l}\right)^{1 / 2}\right)\right) .
$$

Thus

$$
W_{m}=\left(1+O\left(\frac{(\log l)^{3 / 2}}{l^{1 / 2}}\right)\right)\left(\frac{\Delta}{2 \pi l N(1-N)}\right)^{1 / 2} x^{\gamma} V_{m}
$$

where

$$
V_{m}=\exp -\left((m-M k)^{2} / \delta^{2} k\right)
$$

And, therefore,

$$
\sum_{a \leq m<b} W_{m}=\left(1+O\left(\frac{(\log l)^{3 / 2}}{l^{1 / 2}}\right)\right)\left(\frac{\Delta^{3}}{2 \pi l N(1-N)}\right)^{1 / 2} x^{\gamma} \sum_{a \leq m<b} V_{m}
$$

To estimate this sum, we divide the interval $[a, b)$ into $2 q+1$ intervals, each of length $2 L$ :

$$
\sum_{a \leq m<b} V_{m}=\sum_{-q \leq r \leq q} \sum_{m \in I_{r}} V_{m}
$$

where $I_{r}=[M l+(2 r-1) L, M l+(2 r+1) L)$ is the half-open interval of length $2 L$ centered at $c_{r}=M l+2 r L$.

We shall need the following approximation property of $V_{m}$. If $m$ and $c$ are each of the form

$$
m, c=M l+O\left((l \log l)^{1 / 2}\right),
$$

and $|m-c| \leq L$, then we have

$$
\left|\frac{m}{l}-\frac{c}{l}\right|=O\left(\frac{1}{l}\right)
$$

Furthermore, since $(d / d m) \log V_{m}=O(|\mu-M|)$ in a fixed neighborhood of $M$, we have

$$
V_{m}=V_{c}\left(1+O\left(\frac{(\log l)^{1 / 2}}{l^{3 / 2}}\right)\right)
$$

Using this approximation property, we may replace the summand $V_{m}$ by the constant $V_{c_{r}}$ in the inner sum over $m \in I_{r}$, so that

$$
\sum_{m \in I_{r}} V_{m}=\left(1+O\left(\frac{(\log l)^{1 / 2}}{l^{3 / 2}}\right)\right) V_{c_{r}} \sum_{m \in I_{r}} 1
$$


By the Kronecker-Weyl theorem, we have

$$
(1-\varepsilon) \varepsilon 2 L \leq \sum_{m \in I_{r}} 1 \leq(1+\varepsilon) \varepsilon 2 L
$$

for each $r$, since the lucky values of $m$ in $I_{r}$ correspond to lucky values of $i$ in an interval of length $t$. Thus we have

$$
(1-\varepsilon) \varepsilon 2 L \sum_{-q \leq r \leq q} V_{c_{r}} \leq \sum_{a \leq m<b} V_{m} \leq(1+\varepsilon) \varepsilon 2 L \sum_{-q \leq r \leq q} V_{c_{r}} .
$$

The sum $\sum_{-q \leq r \leq q} V_{c_{r}}$ may now be approximated by an integral, extended to an infinite range of integration, and evaluated by an appropriate substitution, all as in the proof of Lemma 2.3. The result is

$$
\sum_{-q \leq r \leq q} V_{c_{r}}=\frac{1}{2 L}\left(\frac{2 \pi l N(1-N)}{\Delta^{3}}\right)^{1 / 2}+O\left(\frac{1}{l^{4}}\right) .
$$

Working backwards through (3.7), (3.6), and (3.5) yields (3.4).

The formula (3.3) follows from Lemma 3.1, since $C \gamma=1 / \Delta$. We observe that the same method works to establish the asymptotic formula (1.7) for $h^{\prime}(x)$ in the irrational case.

Though we have derived (1.4) and (1.5) by parallel arguments, there is an important difference between these derivations. We could have done the analysis in $\S 2$ to obtain an $O$-estimate for the error in (1.5); the most straightforward way of doing this yields a factor of $\left(1+O\left((\log \log x)^{3 / 2}(\log x)^{-1 / 2}\right)\right)$. No such sharpening is possible for (1.4), however, since the Kronecker-Weyl theorem, in the form we have cited, gives no estimate for the rate of convergence to the uniform distribution. The same phenomenon arises for the analytic proof using the Wiener-Ikehara theorem, for while convergence follows from the behavior of $K(s)$ on the critical line and the right half plane it bounds, the rate of convergence depends on how closely the poles in the left half plane approach the critical line as their imaginary parts grow (see [W2], [I], $[\mathrm{L}]$ ). With either method, the missing information depends on how well the irrational number $\log \alpha / \log \beta$ can be approximated by rational numbers as the denominators of these rational numbers grow. This is the crux of the difference: all rational numbers are alike, but each irrational number is irrational in its own way.

Since we have not made any quantitative hypothesis concerning the irrationality of $\log \alpha / \log \beta$, we cannot expect to draw any conclusion about the rate of approach in (1.4). If however we assume that $|\log \alpha / \log \beta-p / q|$ is bounded away from zero by a function of $q$, the elementary method used here (as well as the analytic method used by Fredman and Knuth) can be adapted to yield an explicit $O$-estimate in (1.4).

4. Conclusion. After deriving (1.4) and (1.5) in a new way, and obtaining explicit descriptions of the functions $D(x)$ and $D^{\prime}(x)$ appearing in (1.5) and (1.8), we shall exhibit in this section the consequences of these explicit descriptions for the original recurrence (1.1).

Fredman and Knuth show, by elementary arguments, that

$$
M(n)=1+(\alpha+\beta-1) W(n),
$$

where $W(n)$ is the sum of the weights of the $n$ words having the smallest weights. (Recall that the weight of a word over $\{\alpha, \beta\}$ is the product of its letters.) By the definitions of $h(x)$ and $h^{\prime}(x)$, we have $W(h(x))=h^{\prime}(x)$. Let us assume that $\log \alpha / \log \beta$ 
is rational. Recall that a value of $x$ is "magic" if $x=\varrho^{l}$ for some natural number $l$. We have $D(x)=P(0)$ and $P^{\prime}(x)=P^{\prime}(0)$ for all magic values of $x$, and the asymptotic formulas

$$
h(x) \sim P(0) x^{\gamma}
$$

and

$$
h^{\prime}(x) \sim P^{\prime}(0) x^{\gamma+1}
$$

valid for magic values of $x$.

Let us say that a value of $n$ is "magic" if $n=h(x)$ for some magic value of $x$. Then (4.2) and (4.3) yields the asymptotic formula

$$
W(n) \sim P^{\prime}(0)\left(\frac{n}{P(0)}\right)^{1+1 / \gamma}
$$

valid for magic values of $n$.

To extend (4.4) to arbitrary values of $n$, we observe that as $n$ increases between magic values, $W(n)$ increases by the addition of equal weights. Thus the points of the graph of $W(n)$ between magic values of $n$ lie on the chords joining the points of the graph at successive magic values, and the formula for arbitrary $n$ is obtained by linearly interpolating between the values given by (4.4) for magic values of $n$. This gives

$$
W(n) \sim P^{\prime}(0) Q\left(\left\{\log _{\sigma}\left(\frac{n}{P(0)}\right)\right\}\right)\left(\frac{n}{P(0)}\right)^{1+1 / \gamma},
$$

where $Q(\lambda)=(1-\lambda+\lambda \tau) \tau^{-\lambda}$, which establishes (1.10) with

$$
B(n)=(\alpha+\beta-1) P^{\prime}(0) Q\left(\left\{\log _{\sigma}\left(\frac{n}{P(0)}\right)\right\}\right)\left(\frac{1}{P(0)}\right)^{1+1 / \gamma}
$$

which is periodic in $\log n$ (with period $\log \sigma$ ), as claimed.

We have dealt in this paper with particular recurrences, (1.1) and (1.2), taken from Fredman and Knuth. It is possible to extend the analysis straightforwardly to a number of other recurrences of similar form, as, for example, with initial conditions imposed on an initial segment $\{0,1, \ldots, r\}$ of the domain, rather than just at the point zero, or with three terms on the right-hand side, rather than just two. We see the contribution of this paper, however, as residing more in its methods than in their scope. The Wiener-Ikehara-Landau theorem used by Fredman and Knuth is of an essentially "Tauberian" character, inferring the asymptotics of a sequence from that of its sum. It is virtually equivalent in depth to the prime number theorem, which was in fact the application that motivated Wiener, Ikehara, and Landau. The arguments used in this paper, however, are not only elementary, but also "direct" or "Abelian" in character: they infer the asymptotics of a sum from that of its terms. These arguments are much less delicate than the ones they replace, and they show the phenomena we have studied to be less deep than has hitherto been supposed.

\section{REFERENCES}

[FK] M. L. Fredman AND D. E. KNUTH, Recurrence relations based on minimization, J. Math. Anal. Appl., 48 (1974), pp. 534-559. 
[I] S. IKEHARA, An extension of Landau's theorem in the analytical theory of numbers, J. Math. Phys., 10 (1931), pp. 1-12.

[K1] D. E. KNUTH, The Art of Computer Programming-Volume 1: Fundamental Algorithms, Addison-Wesley, Reading, MA, 1968.

[K2] L. KRONECKER, Näherungsweisse Ganzzahlige Auflösung Linearer Gleichungen, Sitzungsber. Berliner-Akad. Wiss., 46 (1884), pp. $1071 \mathrm{ff}$.

[L] E. LANDAU, Über den Wienerschen neuen Weg zum Primzahlsatz, Sitzber. Preussische Akad. Wiss., (1932), pp. 514-521.

[N] I. Niven, Irrational Numbers, Mathematical Association of America, Washington, D.C., 1956.

[P] N. PIPPenger, An Elementary Approach to Some Analytic Asymptotics, Proc. Scand. Workshop on Algorithm Theory, 3 (1992), pp. 53-61.

[W1] H. WEYL, Über die Gleichverteilung von Zahlen modulo Eins, Math. Annalen, 77 (1916), pp. 313-352.

[W2] N. WIEnER, A new method in Tauberian theorems, J. Math. Phys., 7 (1928), pp. 161-184. 